\title{
Propuesta de interacción entre el verbo y la preposición en la codificación de los eventos de desplazamiento. Estudio contrastivo entre el español y el chino mandarín
}

\section{Resumen}

El español y el chino mandarín han sido clasificados respectivamente como lengua de marco verbal y lengua de marco satélite (Talmy: 1991, 2000). Sin embargo, en el español, los verbos de Manera sí pueden darse con hacia y hasta, y un grupo reducido de ellos lo puede hacer con $a$. La discusión tipológica sobre el chino mandarín se centra en la estructura serial de "verbo de Manera + palabra de Trayectoria", en la que el segundo constituyente que carece de variación morfológica también puede funcionar independientemente como verbo de Trayectoria. Para intentar arrojar luz sobre estas discusiones, se propone que el esquema nuclear — la Trayectoria Direccional - del evento de desplazamiento se genera mediante la interacción entre el verbo y la preposición, en la que el verbo de Manera con movimiento externo implica una Trayectoria No Direccional que desempeña un papel primordial, mientras que la preposición y el segundo constituyente de la estructura serial china solo son indicadores de dirección que no aportan ninguna Trayectoria. El análisis de la combinatoria entre los verbos de Manera y hacia, hasta, $a$, así como sus expresiones equivalentes chinas, apoyan nuestra hipótesis y permiten concluir que: $a$ es locativa; la estructura serial china pertenece al tipo de lengua de marco satélite; el valor extensivo de hasta no favorece la generación de la Trayectoria Direccional, pero el de su equivalente chino sí lo puede hacer.

\section{Palabras clave}

Tipología lingüística, contrastividad español-chino mandarín, evento de desplazamiento, verbo de Manera, preposición.

\begin{abstract}
Spanish and Mandarin Chinese have been classified as verb-framed language and satellite-framed language respectively (Talmy: 1991, 2000). However, in Spanish, manner verbs are compatible with hacia and hasta, and a small group of them can do
\end{abstract}


so with $a$. The typological discussion on Mandarin Chinese focuses on the serial verb construction of "manner verb + path word", in which the second constituent lacking morphological variation can also function independently as path verb. To try to shed light on these discussions, we propose that the core scheme - the directional path - of the motion event is generated through the interaction between the verb and the preposition, in which the manner verb with external movement implies a nondirectional path which plays a primary role, while the preposition and the second constituent of the Chinese serial verb construction are only direction indicators that do not contribute any path. The analysis of the combinatory between manner verbs and hacia, hasta, a, as well as their equivalent Chinese expressions, support our hypothesis and they let conclude that: $a$ is locative; Chinese serial verb construction belongs to the satellite-framed language type; the extensive value of hasta does not favor the generation of the directional path, but that of its Chinese equivalent can do so.

\section{Keywords}

Linguistic typology, Spanish-Mandarin Chinese contrastivity, motion event, manner verb, preposition.

\section{Introducción ${ }^{1}$}

La tipología binaria de lengua de marco verbal y lengua de marco satélite planteada por Talmy $(1991,2000)$ ha sido muy influyente en las últimas tres décadas para estudiar cómo los hablantes de diferentes lenguas conceptualizan y estructuran de una u otra forma el evento de desplazamiento. De acuerdo con este autor, el español es una lengua representante de marco verbal que codifica Trayectoria en el verbo, y el chino mandarín (en adelante, el chino), una lengua representante de marco satélite que codifica Trayectoria en el satélite.

Sin embargo, en el español está ampliamente observado el patrón de lengua de marco satélite en este tipo de eventos, ya que los verbos de Manera generalmente pueden combinarse con los SPs encabezados por las preposiciones hacia y hasta. La

1 Se ha presentado en "The 24th Annual Ohio State University Congress on Hispanic and Lusophone Linguistics (OSUCHiLL) Virtual Event" (12-13 de marzo de 2021) un paper que ha tratado aspectos de la presente investigación no desarrollados aquí. 
polémica en el español ha girado y gira en torno al complemento télico encabezado por la preposición a que, por un lado, no puede combinarse con la mayoría de los verbos de Manera, y por otro, sí es compatible con un grupo reducido de verbos de Manera, como correr y volar.

La discusión sobre la adscripción tipológica del chino se centra en la estructura serial predominante de " $\mathrm{C} 1$ (constituyente 1: verbo de Manera) + C2 (constituyente 2, que codifica Trayectoria)", en la que C2 que carece de variación morfológica también puede funcionar independientemente como verbo de Trayectoria. Talmy (2000) y Shi (2014) consideran que C2 es un complemento direccional, por lo que el chino es una lengua de marco satélite; Tai (2003) argumenta que C2 constituye el núcleo de la predicación semántica y el chino es una lengua de marco verbal; Slobin (2004) y Talmy (2012) estiman que C2 es un verbo y adscriben el chino a un tercer tipo de lengua: lengua de marco equipolente.

Para intentar arrojar luz sobre estas discusiones, proponemos que en la combinatoria de "verbo de Manera + hacia / hasta / a" y sus expresiones correspondientes chinas, el verbo de Manera con movimiento externo (en terminología de Morimoto, 2001) implica una Trayectoria No Direccional, ignorada en la tradición tipológica de Talmy, pero que, de hecho, desempeña un papel primordial en la codificación de un evento de desplazamiento, mientras que las preposiciones españoles y chinas así como C2 de la estructura serial china solo son indicadores de dirección que codifican Dirección (pero no la Trayectoria Direccional). De esta forma, el esquema nuclear - Trayectoria Direccional - de un evento de desplazamiento se genera a través de la interacción entre los dos constituyentes de dicha combinatoria. Para comprobar esta hipótesis, realizaremos un análisis sobre la combinatoria entre los verbos de Manera y las tres preposiciones españolas, así como las combinaciones correspondientes en el chino.

Nuestro trabajo -que constituye uno de los primeros estudios que contrasta directamente el español con el chino bajo el marco teórico de Talmy-podría explicar las discusiones antes mencionadas en las dos lenguas a partir de una nueva perspectiva. Para ello procedemos de la siguiente manera: en la sección 2, presentamos la tipología binaria de Talmy (1991) y el tipo de lengua de marco equipolente propuesto por Slobin (2004). En las secciones 3 y 4, nos adentramos en la discusión en el español y el chino 
acerca de la adscripción tipológica, así como en las expresiones correspondientes a hacia, hasta y a en el chino. En la sección 5, desarrollamos nuestra propuesta sobre la codificación del evento de desplazamiento. En la sección 6, analizamos la combinatoria entre los verbos de Manera y hacia, hasta y a y las expresiones equivalentes chinas. Finalmente, exponemos los resultados obtenidos y las conclusiones en la sección 7.

\section{Marco teórico: la tipología binaria de Talmy y un tercer tipo de lengua: lengua de marco equipolente}

Talmy $(1985,2000)$ propone que, semánticamente, un evento de desplazamiento se compone de cuatro elementos internos y un co-evento. Los cuatro componentes internos son: 1) Figura, que es el objeto que se mueve o que está localizado en un lugar determinado; 2) Fondo, que es el objeto de referencia en el movimiento; 3) Trayectoria, que es el curso seguido por la Figura respecto al Fondo, y constituye el esquema nuclear de un movimiento; 4) Movimiento, que es la presencia per se en el evento de desplazamiento. Por su parte, el co-evento consta, fundamentalmente, de dos componentes: Manera y Causa. Todos estos elementos semánticos pueden proyectarse en los elementos sintácticos, como verbo, adposición, oración subordinada y lo que se denomina satélite, entre otros. A modo de ilustración, se puede ver cómo en la Figura 1 (ejemplo de Talmy, 2000: 26), The pencil y the table son, respectivamente, la Figura y el Fondo, el verbo rolled codifica el Movimiento y el co-evento Manera, y el satélite off expresa la Trayectoria:

\begin{tabular}{|c|c|c|c|}
\hline The pencil & rolled & off & the table \\
\hline Figura & Movimiento + Manera & Trayectoria & Fondo \\
\hline
\end{tabular}

Figura 1. Componentes semánticos de un evento de desplazamiento

Tomando el componente semántico de Trayectoria como constante, Talmy (1991, 2000) propone que las lenguas más habladas del mundo pueden dividirse en dos tipos: 1) el de lengua de marco verbal, que se caracteriza por fusionar la Trayectoria en 
el verbo y el co-evento en un satélite o un adjunto. Pertenecen a este tipo la familia romance, semítica, japonesa, tamil, entre otras; 2) el de lengua de marco satélite, que se caracteriza por codificar la Trayectoria en el satélite y el co-evento en el verbo. Lenguas representantes de este último tipo son la mayoría de las lenguas indoeuropeas menos las lenguas romances, el chino, las lenguas ugrofinesas, etc.

Como ejemplo, se contrasta el comportamiento de codificación de los eventos de desplazamiento entre el español — representante de lengua de marco verbal- $-\mathrm{y}$ el inglés — representante de lengua de marco satélite-

\section{(1) a. The bottle floated out. \\ b. La botella salió flotando.}

(Talmy, 1991: 487)

En (1a), la Trayectoria se expresa en el satélite out y la Manera en el verbo floated, propio de la lengua de marco satélite; en cambio, en (1b) la Trayectoria está fusionado en el verbo salió y la Manera se expresa a través del gerundio flotando, característico de la lengua de marco verbal.

Basándose en la tipología de Talmy, Slobin (2004) propone un tercer tipo de lengua: lengua de marco equipolente, en el que el constituyente que codifica Trayectoria y el que codifica Manera gozan del mismo estatus gramatical, y adscribe a este tipo el chino que rige la serialización verbal, como veremos en la sección 4.

\section{Discusiones sobre el patrón de lengua de marco satélite en el español}

\subsection{El español no dispone de predicado secundario télico}

Aunque el español ha sido clasificado como una lengua de marco verbal, la coexistencia del patrón de lengua de marco satélite ${ }^{2}$, como vemos con los siguientes ejemplos de Aske (1989), vulnera la tipología de Talmy:

2 Cabe señalar que Talmy (2000) excluye los SPs de la categoría de satélite. No obstante, como el SP en la combinatoria de "verbo de Manera + SP", totalmente contradictoria con el patrón de lengua de marco verbal, se encarga de codificar la Trayectoria, consideramos que el SP tiene un comportamiento afín al satélite y por ello tomamos esta combinatoria como un patrón válido de lengua de marco satélite (También véase Beavers et al. (2010: 346) sobre "verbo de Manera + hasta"). 
(2) a. Juan bailó en círculos / de un lado para otro / hacia la puerta / hasta la puerta.

b. La botella flotó hacia la cueva.

c. El libro se deslizó hasta el suelo.

d. La pelota rodó desde el tercer piso hasta el segundo.

e. La botella flotó por el canal.

f. El globo flotó para arriba, para abajo, para adentro y luego para afuera.

(Aske, 1989: 3)

En todas estas construcciones, el verbo codifica la Manera y la Trayectoria se representa por un SP, demostrando así características propias de las lenguas de marco satélite.

No obstante, Aske (1989) apunta que en el español este patrón solo se limita a la estructura de "verbo de Manera + complemento atélico", como demuestran los ejemplos en $(2)^{3}$, mientras que la combinatoria de "verbo de Manera + complemento télico o resultativo" no existe gramaticalmente, ya que el español carece de predicado secundario télico como el del inglés (Blew off the table) y tiene que acudir a un verbo (salió volando de la mesa) para expresar la Trayectoria télica. De esta forma, la aceptabilidad del patrón de lengua de marco satélite en el español se reduce a los complementos atélicos, encabezados por hacia, hasta, desde, por, entre otras.

\section{2. ¿La preposición $a$ es direccional o locativa?}

Con respecto a la telicidad del español, Fábregas (2007) argumenta que en el español la imposibilidad de combinación de algunos verbos de Manera con complementos direccionales se debe a que la preposición $a$, distinta de to y parecida a at del inglés, es locativa que "denotes a place relationship where the figure is in contact with (at least) one point of the boundary of the bound". Esta preposición se diferencia de la locativa en que "expresses a place relationship where the figure is contained in the ground or supported by it”' (Fábregas, 2007: 178).

3 Para Aske (1989), hasta es una preposición atélica que no indica meta (véase el punto 3.4.1). 
Debido al diferente significado de las dos preposiciones locativas, estas se comportan de forma distinta en construcción locativa y direccional en cuanto a tres aspectos. En primer lugar, en contextos que denotan límite o que tengan un significado relacionado con él, se usa la preposición a: Juan está a la entrada de la casa. En segundo lugar, en construcciones locativas el significado de una oración varía según la preposición sea en o a, por ejemplo, en Juan está en/a el piano, con en significa que 'Juan está dentro del piano', mientras que con $a$ literalmente significa que 'Juan está en contacto con algún límite del piano', de ahí que se interprete como que 'Juan está tocando al piano'. En tercer lugar, se prefiere usar la preposición $a$ en construcciones direccionales: El pájaro voló a su nido (que solo tiene significado direccional), frente a \#El pájaro voló en su nido (que no tiene significado direccional). En suma, pese a ser una preposición locativa, el autor concluye que $a$ es la mejor opción para introducir un Fondo locativo en construcciones direccionales para denotar el contacto entre la Figura y el Fondo.

Una vez especificadas las características locativas de $a$, el autor propone que, debido a la codificación de Trayectoria en los verbos de Manera como volar, correr, caminar, su combinación con $a$ es gramatical, mientras que para los verbos que solo denotan un movimiento interno del cuerpo y no implican ninguna Trayectoria, como flotar, temblar, bailar, su combinación con a resulta agramatical ya que en toda la construcción ningún ítem léxico lexicaliza la Trayectoria.

Demonte (2011) refuta el planteamiento de que $a$ sea una preposición locativa, dado que, en primer lugar, en los ejemplos ofrecidos por Fábregas, el SP encabezado por $a$ solo se combina con verbos estativos (Juan está al piano); en segundo lugar, a se comporta diferente que la à locativa de algunas lenguas como, por ejemplo, el francés, donde esta preposición se aplica para indicar lugares de referencia especificados, o, el inglés, donde la preposición at se usa en contexto diferente que $a$ : I will meet you at the airport. / *Te encuentro al aeropuerto; en tercer lugar, a tiene significado direccional, ya que puede formar "estructuras correlativas con adjuntos encabezados por de o desde que son preposiciones con un claro significado de dirección y no de locación" (Demonte, 2011: 36): Fui a Pisa, desde Siena. / Volví a la terraza, desde allí; por otra parte, y lo que es más importante para comprobar su naturaleza direccional, la preposición a puede alternar con hasta para delimitar una meta. 


\subsection{La importancia de la direccionalidad del verbo de movimiento para combinarse con $a$}

Desde la perspectiva de la Gramática de Construcciones, Pedersen (2016) hace una recopilación exhaustiva de un corpus consistente en 249 verbos intransitivos de movimiento y su combinación con el complemento télico encabezado por $a$. Una vez obtenido el corpus, realiza, a través del Fischer's Exact Test, un collostructional analysis ${ }^{4}$ entre el evento de desplazamiento télico y el lexema verbal. Primero, se lleva a cabo el Test y se ordenan los 249 verbos de movimiento en una tabla según el valor $p$ obtenido, el cual consiste en un indicador del grado de asociación entre el significado verbal y la construcción télica que lo abarca. Después, se agrupan estos verbos tomando como referencia el punto de ruptura $p<0,01$, nivel crítico de asociación que separa los verbos caracterizados por "estar asociado con la construcción télica" (cuando el $p$ es menor a 0,01 ) de los que "no están asociados con la construcción télica" (cuando el $p$ es mayor a 0,01$)$.

Según la tabla de ranking obtenida, los 47 verbos totales que poseen un valor $p$ menor a 0,01 comprenden 40 verbos de Trayectoria y verbos de Trayectoria y Manera, así como 7 verbos de Manera que también implican una direccionalidad fuerte: correr, saltar, volar, lanzarse, tirarse, arrojarse, rodar. Además, los primeros 20 verbos que ocupan la tabla de ranking son todos verbos de Trayectoria.

Por otra parte, todos los verbos de Manera de la tabla pueden dividirse en tres grupos en función del valor $p$ obtenido, y presentan un comportamiento distinto en la combinatoria de "verbos de Manera $+a$ ":

1) Available manner verbs: poseen un valor $p$ menor a 0.01 , lo que indica una asociación positiva entre el verbo integrante de este grupo y la construcción télica. Todos los verbos de Manera de este tipo denotan una direccionalidad: Pedro corrió a la playa.

2) Excludable manner verbs: poseen un valor $p$ mayor a 0.01 , lo que indica que los verbos de este grupo no están asociados con la construcción télica o que la correlación es débil. La mayoría de estos verbos de Manera no solo denotan una

4 De acuerdo con Pedersen (2016: 13), "collostructional analysis applies the principles of measuring lexical collocation to the interaction of lexemes and the grammatical constructions associated with them in the internal structure of constructions". 
direccionalidad, sino que también parecen implicar una Manera más destacada que los del grupo anterior. Por otra parte, el uso télico se da ocasionalmente, cuya gramaticalidad a veces es controvertida: ?? Anda al hotel donde yo estoy,... (Citado en Davies, 2002) / ?? Caminó a la biblioteca.

3) Unavailable manner verbs: poseen un valor $p$ casi igual a 1 y no permiten en ningún caso el uso télico. Se refiere fundamentalmente a verbos de Manera que no implican en absoluto la direccionalidad: *Pedro bailó al baño.

De acuerdo con el comportamiento de los 249 verbos de movimiento - de Trayectoria, de Trayectoria y Manera así como de Manera - en la construcción télica, el autor concluye que la construcción de desplazamiento télico se ve muy restringida por el comportamiento semántico del lexema verbal, es decir, que la implicación de direccionalidad en un verbo de movimiento constituye una condición mínima para codificar un evento télico.

\subsection{Discusión sobre la preposición hasta y su diferencia con la preposición a}

\subsubsection{Hasta sí es un indicador de meta}

Aske (1989) considera hasta como una preposición atélica que no indica el lugar final, ya que no puede coaparecer con un adverbio durativo télico: Juan caminó hasta la cima (?* en dos horas). No obstante, esta afirmación fue rebatida por muchos investigadores posteriores (Morimoto, 2001; Cuartero, 2006; Fábregas, 2007, entre otros), quienes coinciden en afirmar que hasta sí denota el límite final de un movimiento. Por dar algunos ejemplos, Cuartero (2006) plantea que el ejemplo dado por Aske tampoco es combinable con un adverbio durativo atélico: *Juan caminó hasta la cima durante dos horas; Fábregas, por su parte, niega que hasta sea atélica como hacia:

(3) a. Juan empezó a correr hacia su casa.

b. *Juan empezó a correr hasta su casa.

(Fábregas, 2007: 171) 
La frase verbal 'empezar a' no puede coaparecer con un complemento télico. En contraste con (3a), la aparición de hasta en (3b) convierte el predicado en un sintagma télico y por eso ilegitima toda la oración.

\subsubsection{Distinción entre hasta y $a$}

Aunque las dos preposiciones indican meta, se distinguen en que hasta también denota un valor extensivo en el espacio y tiempo, que implica la continuación de una acción hasta el término de movimiento (Penas, 2016; Demonte, 2011). Esta diferencia ${ }^{5}$ puede observarse claramente mediante los siguientes ejemplos de Morimoto (2001), en que (4a) resulta agramatical, ya que el punto espacial implicado por el verbo arribar y el espacio extensivo denotado por hasta se oponen:

(4) a. *arribar hasta el puerto.

b. arribar al puerto.

(Morimoto, 2001: 126)

Otro valor distinto de hasta es que suele marcar "la meta 'salient / expected' (que suele coincidir con la Meta real)" (Demonte, 2011: 37):

(5) a. Juan bajó (al sótano, hasta la entrada a la bodega). (Demonte, 2011: 37)

b. El tren llegó hasta Madrid, (* y ahora se va a Sevilla).

c. El tren ha llegado a Madrid, y ahora se va a Sevilla.

En (5a) el SP introducido por hasta indica la meta real del movimiento, modificando al SP encabezado por $a$. La agramaticalidad de (5b) respecto de $(5 \mathrm{c})$ también prueba el valor distinto de hasta.

En esta sección, hemos repasado la discusión sobre la existencia del patrón de lengua de marco satélite en el español. El debate se ha focalizado en la combinatoria de "verbo de Manera + a", en la que la mayoría de los verbos de Manera no pueden darse con $a$, mientras que algunos de ellos sí lo pueden hacer. La preposición $a$ generalmente

5 Como veremos en 4.2, la expresión correspondiente china de hasta, en contraste con la de $a$, también prueba esta diferencia. 
ha sido considerada como un indicador de meta que codifica Trayectoria, mientras que Fábregas (2007) considera que es locativa, por lo que solo se da con los verbos de Manera que codifican Trayectoria; por otra parte, el otro indicador de meta hasta que tiene un valor extensivo adicional que $a$, y el indicador de dirección hacia, pueden darse con casi todos los VMMEs.

\section{Discusión sobre la adscripción tipológica del chino y las expresiones correspondientes a hacia / hasta / a}

\subsection{Discusión sobre la adscripción tipológica del chino}

En el chino la discusión tipológica se centra en la estructura serial que esta lengua rige en la descripción del evento de desplazamiento. Algunos investigadores consideran que la estructura serial pertenece al tipo de lengua de marco satélite, mientras que otros la toman como propia de lengua de marco verbal o de lengua de marco equipolente. Antes de exponer las diferentes opiniones acerca de esta cuestión, es necesario conocer las dos formas de codificación del evento de desplazamiento en el chino:

1) Estructura serial compuesta por $\mathrm{C} 1$ (Constituyente 1: verbo de Manera) $+\mathrm{C} 2$ (Constituyente 2, que codifica Trayectoria) + / - C3 (Constituyente 3, que es la deixis qù 'ir' o lái 'venir'):

\begin{tabular}{|c|c|c|c|c|}
\hline$T \bar{a} \quad$ păo & dào & jiàoshì & $(+/-q \grave{u})$ & le. \\
\hline correr & llegar; a & aula & (+/-deixis-ir) & PP (partícula perfectiva) \\
\hline
\end{tabular}

En (6), el verbo de Manera păo 'correr' que es C1 de la estructura serial, codifica Movimiento y Manera; C2 dào 'llegar; a' codifica Trayectoria; y C3 qù 'ir', que puede aparecer o no en esta oración, codifica la Trayectoria 'en dirección alejada del

6 También se puede traducir como ‘Él corrió (en dirección alejada del hablante) y llegó al aula' o ‘Él llegó corriendo al aula (en dirección alejada del hablante)', dependiendo de si se toma la estructura serial como propia de lengua de marco equipolente o de lengua de marco verbal, respectivamente. Como argumentaremos que $\mathrm{C} 2$ de la estructura serial es un indicador de dirección y que la estructura serial debería pertenecer a la lengua de marco satélite, hemos optado por la traducción correspondiente a este último tipo de lengua. 
hablante'. Como vemos seguidamente, si se consideran C2 y C3 como verbos en esta construcción, estos también codifican Movimiento. En esta oración, también se puede eliminar C2 dào 'llegar; a' y, en este caso, C3 qù 'ir' puede aparecer antes o después del Fondo jiàoshi 'aula', funcionando como C2 de la estructura serial. Para no complicar más de lo necesario la explicación en los siguientes ejemplos, a partir de ahora solo tenemos en cuenta $\mathrm{C} 2$, sabiendo que $\mathrm{C} 3$ también puede funcionar como $\mathrm{C} 2$.

Además de dào 'llegar; a' y qù 'ir', otros verbos de Trayectoria que también pueden funcionar como $\mathrm{C} 2$ son, entre otros, los siguientes:

(7) shàng 'subir', xià 'bajar', jìn 'entrar', chū 'salir', huí 'regresar', guò 'cruzar', lái 'venir', q̌̌ 'alzar', diào 'caer', etc.

2) C2 de la estructura serial, que carece de variación morfológica, puede funcionar independientemente como predicado de la oración:

(8) dào jiàoshì le.
Él llegar aula PP
'Él llegó al aula'

Aunque la segunda forma de codificación, propia de lengua de marco verbal, es totalmente gramatical, la estructura serial es la que predomina en chino en la descripción del evento de desplazamiento (Shi, 2014; Chen, 2019, entre otros.). El estatus gramatical de $\mathrm{C} 2$, como hemos mencionado al principio de este apartado, constituye el foco de debate en cuanto a la adscripción tipológica del chino. En la gramática tradicional, $\mathrm{C} 2$ ha sido considerado como complemento direccional, por lo que Talmy $(1985,1991,2000)$ adscribe el chino al tipo de lengua de marco satélite. Esta visión se ve apoyada por los estudios diacrónicos, según los cuales el chino sufrió una conversión tipológica de lengua de marco verbal a lengua de marco satélite ( $\mathrm{Li}$, 1997; Peyraube, 2006; Shi, 2014), proceso por el que el verbo de Trayectoria del chino antiguo ha adquirido un estatus de complemento, incorporando también un significado aspectual, como ejemplificamos con dào 'llegar; a': 
a. Tā zhōngyú zhăo dào le Bill de zōngjì. (Li, 1997: 242)

él por fin buscar llegar; a PP Bill posesivo paradero

'Por fin él encontró el paradero de Bill'

b. Jingchá bă xiăotōu zhūa dào le.

policía PA ladrón detener llegar; a PP

'La policía ya detuvo al ladrón'

En (9), dào aparece después del verbo zhăo 'buscar' (9a) y zhuā 'detener' (9b) para indicar la completitud de la acción verbal, con la función de complemento aspectual perfectivo.

Sin embargo, Tai (2003) argumenta que, semánticamente, C2 de la estructura serial, al igual que dào en (6), debe ser entendido como el centro de la predicación semántica, ya que de dicha estructura se puede eliminar C1: el verbo de Manera, como vemos en (8), pero no C2 que codifica Trayectoria, como mostramos en (10):

$\begin{array}{lll}* T a \bar{a} \text { păo jiàoshi } & \text { le. } \\ \text { Él correr aula } & \mathrm{PP} \\ \text { *'Él corrió el aula' } & \end{array}$

Por lo tanto, este autor adscribe la estructura serial al tipo de lengua de marco verbal. En nuestro trabajo descartamos esta visión, puesto que en el chino los verbos de Trayectoria implican per se la dirección de desplazamiento y se combinan con el Fondo sin que se intercale ninguna preposición o satélite (a diferencia del español y el inglés), mientras que los verbos de Manera sí necesitan la ayuda de un indicador de dirección para especificar la relación de desplazamiento de la Figura en relación con el Fondo (al igual que el español y el inglés); en otras palabras, el método de eliminación adoptado por Tai (2003) para examinar el estatus semántico de los constituyentes de la estructura serial es inadecuada e inexacta.

Como hemos mencionado en la sección 2, Slobin (2004) propone que C1 y C2 son ambos verbos y que el chino pertenece al tipo de lengua de marco equipolente. En relación con esto, Talmy (2012) apunta que el patrón de marco equipolente se limita a la estructura serial compuesta por el verbo de Manera y cualquiera de los siguientes 
doce verbos de Trayectoria que mantienen el mismo significado, tanto cuando estos aparecen como $\mathrm{C} 2$ de la estructura serial como cuando funcionan como verbo independiente:

(11) shàng 'subir', xià 'bajar', jìn 'entrar', chū 'salir', huí 'regresar', guò 'cruzar', dào 'llegar', dăo 'derrocar', kāi 'abrir', zǒu 'alejarse', qù 'ir', lái 'venir'.

(Talmy, ibid.: 17)

Por otra parte, existe otro grupo de C2, compuesto por al menos catorce términos, que, cuando funciona independientemente como verbo, carece de correspondencia con C2 en alguno de los siguientes casos: 1) cuando $\mathrm{C} 2$ no puede funcionar como predicado; 2) cuando, aunque pueda funcionar independientemente como verbo, su significado ya difiere del de $\mathrm{C} 2$, porque tiene otro significado de Trayectoria o un significado no relacionado con la Trayectoria; y 3 ) cuando funciona independientemente como verbo, pero solo conserva un mínimo significado de C2 en usos muy restringidos:

(12) Kāi 'alejarse', yuăn 'alejarse', păo 'alejarse', fēi 'alejarse de un punto', jìn 'hacia', zháo 'a un punto de contacto', zhù 'a un punto de una superficie, con resistencia a movimiento que se aleja de este punto', q̌̃ 'hacia arriba', diào 'caer', fān 'al revés', sàn 'dispersar', chéng 'radialmente hacia dentro', zhòng 'dar en el blanco', măn 'lleno'.

(Talmy, ibid.: 18)

Se puede ilustrar con el verbo kāi, el cual, cuando funciona como verbo de Trayectoria, significa 'abrir' (13a) o 'conducir' (13b), pero cuando aparece en la estructura serial (13c), aunque mantiene la misma forma morfológica, ya no tiene este significado sino el de ‘lejos, hacia lejos' equivalente al satélite away del inglés:
(13) a. $k \bar{a} i$ mén
abrir puerta
'abrir la puerta'
b. $\boldsymbol{k} \bar{a} \boldsymbol{i} \quad \mathrm{che}$ 


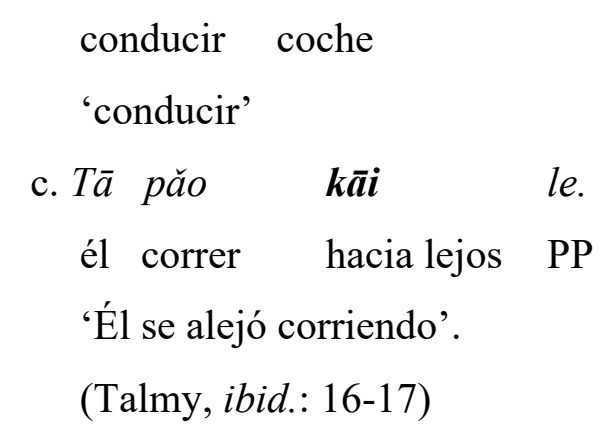

En suma, en este apartado hemos expuesto las razones por las cuales se considera respectivamente la estructura serial del chino como lengua de marco satélite, lengua de marco verbal y lengua de marco equipolente, y hemos descartado la opinión de Tai (2003) referente a que la estructura serial pertenece al tipo de lengua de marco verbal. En la sección 6, argumentaremos que C2 funciona como complemento direccional y que la estructura serial china debería pertenecer al tipo de lengua de marco satélite.

\subsection{La expresión china de las preposiciones hacia, hasta y a}

En el chino las preposiciones que expresan el significado de hacia son principalmente xiàng, cháo o wăng, siendo la primera la más frecuentemente utilizada. El SP encabezado por estas preposiciones suele anteponerse al verbo de Manera:

$\begin{array}{lllll}\text { (14) } T a \bar{a} \text { xiàng / cháo / wăng jiàoshì păo } & \text { le. } \\ \text { Él hacia } & \text { aula correr } & \text { PP } \\ \text { 'Él corrió hacia el aula' } & & & \end{array}$

La preposición $a$ se expresa con la palabra dào, que, como hemos observado en el apartado anterior, puede funcionar como verbo independiente con el significado de 'llegar' (8), seguido directamente de un lugar de destino, o puede aparecer después de un verbo de Manera para formar una estructura serial (6).

En el chino no existe una preposición específica para expresar el significado de hasta en los eventos de desplazamiento, por lo que se acude a una forma analítica añadiendo antes de la estructura serial 'verb de Manera + dào' un adverbio yizhí (literalmente yì significa 'uno' y zhí, 'recto'), que significa 'continuamente, todo recto' 
(DCM, 2016). Por lo tanto, para expresar 'él corrió hasta el aula', la estructura correspondiente es:
(15) Tā yìhí
păo dào le jiàoshì.
Él continuamente correr llegar; a PP aula
'Él corrió hasta el aula'

\section{Tipo de evento de desplazamiento y verbo de movimiento objeto de estudio. Nuestra hipótesis}

En nuestro modelo de descripción, nos centramos en el evento de desplazamiento no agentivo, que describe el desplazamiento espacial de la Figura de un lugar a otro. Proponemos que el esquema nuclear de un evento de desplazamiento debe ser Trayectoria Direccional (TD) en vez de Trayectoria, ya que este tipo de evento siempre implica una Dirección ${ }^{7}$, a diferencia de un evento de desplazamiento no direccional que no implica ninguna dirección de movimiento, como en Juan caminó en el parque, donde la Figura 'Juan' se desplaza en el parque generando una Trayectoria No Direccional (TND), donde no se especifica la Dirección del desplazamiento.

En lo que respecta a los verbos de movimiento, nos basaremos principalmente en la clasificación de Morimoto (2001) y analizaremos tres tipos de verbos de Manera (intransitivos):

1) verbos de Manera con movimiento de referencia externa (VMME), como andar, arrastrarse, caminar, correr, deslizarse, gatear, nadar, etc.

2) verbos de Manera con movimiento interno (VMMI), como agitarse, balancearse, bambolearse, ondear, tambalearse, temblar, patalear, etc.

3 ) verbos de Manera estática $(\mathrm{VME})^{8}$, como reír, cantar, enfadarse, etc.

7 De esta manera, lo que codifican los verbos de Trayectoria no es sino Trayectoria Direccional. De hecho, esta afirmación está en línea con muchos trabajos de autores precedentes. Así, Levin (1993) considera que los verbos de Trayectoria implican inherentemente una Dirección; Wälchli (2001), Berthele (2004) y Cifuentes (2008) clasifican los verbos de Trayectoria en función del tipo de Dirección que señalan.

8 El verbo de Manera estática es acuñación nuestra, mientras que las de los otros dos verbos de Manera son de Morimoto (2001). 
En cuanto a los VMMEs, algunos investigadores han apuntado previamente la existencia de Trayectoria en ellos. Lamiroy (1991) observa que los VMMEs implican un desplazamiento, que "no se hace por referencia a un punto determinado por la posición del hablante y por la geometría del espacio" (Lamiroy, 1991: 66). Siguiendo esta opinión, Morimoto (2001) advierte que estos verbos de Manera "se limitan a señalar la existencia de un desplazamiento, sin concretar, a nivel léxico, qué tipo de Trayectoria está implicado en dicho desplazamiento" (Morimoto, 2001: 46). En el chino, Fan (2013: 106) menciona que cuando un verbo de movimiento expresa el concepto de Movimiento, también ha de existir en él una Trayectoria inherente. Basándonos en las opiniones anteriores, proponemos que el VMME implica una TND, ya que su acción verbal siempre denota el desplazamiento de la Figura en el mundo físico. En cambio, el VMMI y el VME no denotan ningún desplazamiento de la Figura, por eso no implican ningún tipo de Trayectoria.

Además de los verbos de Manera, en el punto 6.3 ““"verbo + $a$ ” en español” también vamos a tener en cuenta el verbo de $T D$, como ir, venir, entrar, salir, subir, bajar, alejarse, etc., que implica una $T D$.

Proponemos que, en la codificación del evento de desplazamiento, la TND implicada en el verbo juega un papel primordial, mientras que las preposiciones no codifican per se ningún tipo de Trayectoria y solo funcionan como indicadores de dirección que codifican Dirección. La interacción entre el verbo y la preposición genera la TD del evento de desplazamiento.

\section{Interacción entre el verbo y la preposición en la generación de la TD}

Con el fin de comprobar la hipótesis que acabamos de plantear, en esta sección procedemos a analizar cómo se genera la TD del evento de desplazamiento en la combinación entre los verbos y las preposiciones hacia, hasta, a, y sus expresiones correspondientes chinas. En 6.1, vamos a estudiar dichas combinatorias en el chino. En 6.2, analizamos la combinatoria entre los tres tipos de verbos de Manera con hacia / hasta, y en 6.3, la combinatoria entre los verbos y la preposición polémica $a$. Nuestra propuesta podría aportar luz a las discusiones más críticas para el español y el chino; 
en concreto, en lo que respecta a la propuesta que hacemos de que la agramaticalidad entre la mayoría de los VMMEs y $a$ se deba a que $a$ es locativa, y a que C2 de la estructura serial china sea un indicador de dirección y la estructura serial a la que pertenece C2 sea del tipo de lengua de marco satélite.

\section{1. la expresión correspondiente de "verbo de Manera + hacia / hasta / a" en el chino}

Cuando el verbo pertenece a un VMME, como en (16), puede darse con xiàng9 'hacia' (16a), o con el marco preposicional yizhí + V + dào 'V + hasta' y dào 'llegar; a' (16b) sin restricciones:

(16) a. xiàng 'hacia' + gángkǒu 'puerto' + [pǎo 'correr' / fēi 'volar' / qí 'cabalgar' / sànbù 'pasear' / quéxíng 'cojear'].

'correr / volar / cabalgar / pasear / cojear hacia el puerto'

b. [+/- yìzhí 'continuamente'] + [păo 'correr' / fềi 'volar' / qí 'cabalgar' / sànbù 'pasear' / quéxíng 'cojear'] + dào 'llegar; a' + gángkǒu 'puerto'.

'correr / volar / cabalgar / pasear / cojear a / hasta el puerto'

La generación de la TD en dichas combinatorias puede atribuirse a alguna de las siguientes posibilidades:

1) el VMME implica una TND y la preposición xiàng 'hacia', C2 dào 'llegar; a' y el marco preposicional yìzhi $+\mathrm{V}+$ dào 'hasta $+\mathrm{V}$ ' implican una TD. Cualquier Trayectoria, es decir, la TND del VMME o la TD de xiàng 'hacia', C2 dào 'llegar; a' y el marco preposicional yizhí $+\mathrm{V}+$ dào 'hasta $+\mathrm{V}$ ', puede funcionar para codificar una TD (en combinación con la Dirección implicada por xiàng 'hacia', C2 dào 'llegar; a' y el marco preposicional yìzhí + V + dào 'hasta + V', en el primer caso);

2) el VMME implica una TND y xiàng 'hacia', C2 dào 'llegar; a' y el marco preposicional yìzhí $+\mathrm{V}+$ dào 'hasta $+\mathrm{V}$ ' son indicadores de dirección que solo codifican Dirección; la combinación entre ellos genera la TD del evento de desplazamiento;

9 En el análisis solo vamos a tener en cuenta la preposición más frecuentemente adoptada xiàng 'hacia'. 
3) el VMME no implica TND y xiàng 'hacia', C2 dào 'llegar; a' y el marco preposicional yìzhi $+\mathrm{V}+$ dào 'hasta $+\mathrm{V}$ ' implican una TD. La TD implicada en las últimas constituye la TD del evento de desplazamiento. Esta posibilidad coincide básicamente con la propuesta de Talmy (1991, 2000), según la cual, el VMME codifica Movimiento, y el resto, la Trayectoria (TD, en nuestra propuesta).

Cuando se sustituye el VMME por el VMMI (17a-17b) o el VME (17c-17d) que no implica TND, no puede combinarse con xiàng 'hacia' (17a y 17c) y dào 'llegar; a' (17b y $17 d)$ para generar la TD:

a. *xiàng 'hacia' + jiàoshì 'aula' + [chàndǒu 'temblar' / zhèndòng 'estremecerse' / kebàn 'tropezar' / yáobăi 'agitarse' / yáotóu 'cabecear' / dū̄suo 'tiritar' / wānqqū 'curvarse' / juăn 'enrollarse' / lāshēn 'estirarse' / duòjiăo 'patalear' / xuánzhuăn 'girar'].

*‘Temblar / estremecerse / tropezar / agitarse / cabecear / tiritar / curvarse / enrollarse / estirarse / patalear / girar hacia el aula'

b. *[chàndǒu 'temblar' / zhèndòng 'estremecerse' / kebàn 'tropezar' / yáobăi 'agitarse' / yáotóu 'cabecear' / duōsuo 'tiritar' / wānqū 'curvarse' / juăn 'enrollarse' / lāshēn 'estirarse' / duòjiăo 'patalear' / xuánzhuăn 'girar'] + dào 'llegar; a' + jiàoshì 'aula'

*`Temblar / estremecerse / tropezar / agitarse / cabecear / tiritar / curvarse / enrollarse / estirarse / patalear / girar al aula'

c. *Xiăomíng xiàng jiàoshì xiào/kü/chàng/qì le.

Xiaoming hacia aula reír/llorar/cantar/enfadarse PP

*'Xiaoming se rió / lloró / cantó / se enfadó hacia el aula'

d. *Xiăomíng xiào/kü/chàng/qì dào le jiàoshì.

Xiaoming reír/llorar/cantar/enfadarse llegar; a PP aula

*'Xiaoming se rió / lloró / cantó / se enfadó al aula'

La agramaticalidad de estos ejemplos muestra que la TND implicada en el verbo de Manera es imprescindible en la generación de la TD, ya que cuando este verbo no puede aportar ningún tipo de Trayectoria, xiàng 'hacia' y dào 'llegar; a' no pueden aportar el significado de Trayectoria para que la acción verbal de movimiento interno 
o estática se realice en el espacio según la dirección indicada por ellos mismos y se genere la TD. Este resultado, coincidente con la posibilidad 2) que acabamos de exponer, es totalmente aplicable a otros verbos de TD (véase (11)) que también pueden funcionar como $\mathrm{C} 2$ en la estructura serial. El comportamiento subordinado de C2 al VMME precedente muestra que $\mathrm{C} 2$ solo funciona como un indicador de dirección en la estructura serial, por lo tanto, la estructura serial china debería ser del tipo de lengua de marco satélite.

Sin embargo, aunque el marco preposicional yizhí $+\mathrm{V}+$ dào 'hasta $+\mathrm{V}$ ' no puede darse con muchos VMMIs, sí lo puede hacer con algunos de ellos (18a), así como con los VMEs (18b):
a. $T \bar{a}$ yìzhí
duōsuo dào
le jiàoshi.
él continuamente tiritar llegar; a PP aula
'Él se fue tiritando hasta el aula'
b. Tã yìzhí xiào/kü/chàng/qì dào le jiàoshì.
él continuamente reír/llorar/ cantar/enfadarse llegar; a PP aula
'Él (se) fue riéndo(se) / llorando / cantando / enfadándose hasta el aula'

Consideramos que la gramaticalidad de dichos ejemplos, en contraste con la agramaticalidad de la combinatoria entre los VMMEs o VMEs y dào 'llegar; a', se debe al valor extensivo del marco preposicional, que supone la única diferencia con dào 'llegar; a', el cual es capaz de indicar la realización de la acción verbal de movimiento interno o estática a lo largo del espacio, generando así una trayectoria según la dirección indicada por el marco preposicional.

\section{2. "Verbo de Manera + hacia / hasta" en el español}

Al igual que el chino, los VMMEs en español normalmente no tienen restricciones para combinarse con los SPs introducidos por hacia y hasta:

(19) [Corrió / voló / nadó / caminó / flotó / navegó / cabalgó / callejeó] + [hacia / hasta] la escuela. 
Sin embargo, VMMEs como vagar, cojear, renquear sí presentan ciertas restricciones a la hora de combinarse con hacia y hasta, como vemos en los siguientes ejemplos:

(20) [?vagó / ?cojeó / ?renqueó] + [hacia / hasta] el puerto.

Esto parece deberse a que el uso prevalente del patrón de lengua de marco verbal tiene como consecuencia restringir el desarrollo del de lengua de marco satélite. El hecho de que se prefiera acudir a la forma de gerundio de los VMMEs o frases adverbiales para describir la Manera de un desplazamiento (por ejemplo: se fue cojeando hacia Madrid), hace que la combinatoria de "VMME + hacia" con los VMMEs que denotan una Manera marcada y menos movilidad (TND menos activa), o que son de uso menos frecuente, se encuentre en un estado menos desarrollado, y consiguientemente, resulte extraño o anómalo.

Cuando el verbo es VMMI (21a y 21b) o VME (21c) que no implican ningún tipo de Trayectoria, su interacción con hacia y hasta no permite generar la TD en español:

(21) a. *Juan [tembló / se agitó / se estremeció / osciló / titubeó / tropezó / pataleó / cabeceó / pirueteó $]+[$ hacia / hasta] la puerta.

b. *El animal [se enrolló / se curvó] + [hacia / hasta] la puerta.

c. *Juan [se rió / lloró / cantó / se enfadó] + [hacia / hasta] la puerta.

La agramaticalidad de estos ejemplos prueba que, por un lado, al igual que en chino, en el español la TND implicada en el verbo es fundamental para generar la TD del evento de desplazamiento, y las preposiciones se comportan como indicadores de dirección que solo codifican Dirección; por otro lado, a diferencia del marco preposicional chino yizhí $+\mathrm{V}+$ dào 'hasta $+\mathrm{V}$ ', el valor extensivo de hasta es incapaz de indicar la realización del movimiento interno o la acción estática en el espacio cuando el verbo no implica per se TND.

No obstante, existe un grupo de verbos considerado como VMMI que puede darse con hacia y hasta en el español y las expresiones correspondientes chinas, así como 
con dào 'llegar; a' en el chino, como en bailar y tambalearse (22) y los verbos correspondientes chinos (23):

(22) a. Bailé hacia la ventana, mostrando algunos movimientos sexys mientras daba vuelta alrededor de la mesa. (ETT)

b. Bailó hasta su asiento haciendo el famoso paso lunar de Michael Jackson de espaldas. (ETT)

c. Se tambaleó hacia el bosque y encontró refugio. (ETT)

d. Se tambaleó hasta la cocina. (ETT)

$$
\begin{aligned}
& \text { a. Tā cháo chēzhàn yáohuàng/tiào qù le. } \\
& \text { él hacia estación tambalear/bailar deixis-ir PP } \\
& \text { 'Él se tambaleó/bailó hacia la estación (en dirección alejada del hablante)' } \\
& \text { b. Tā yáohuàng/tiào dào chēzhàn qù le. } \\
& \text { él tambalear/bailar llegar; a estación deixis-ir PP } \\
& \text { 'Él se fue tambaleándo(se)/bailando a la estación (en dirección alejada del } \\
& \text { hablante)' }
\end{aligned}
$$

Por su parte, Fábregas (2007) afirma que hacia y hasta codifican una Trayectoria ( $T D$ en nuestra terminología) y por eso pueden darse con estos VMMIs que no la implican.

Sin embargo, si dichos indicadores de dirección implicaran una TD, deberían poder combinarse con los VMMIs y VMEs sin dificultad para generar la TD del evento de desplazamiento. Como se muestra en (17) y (21), en el español y el chino los VMMIs y VMEs generalmente son incompatibles con estos indicadores de dirección. Por otra parte, los VMMIs españoles que pueden darse con hacia y hasta básicamente coinciden con los chinos que pueden darse con xiàng 'hacia' y dào 'llegar; a', como bailar, tambalearse, flotar, rebotar y tiào 'bailar', yáohuàng 'tambalearse', piāo 'flotar', tán 'rebotar', entre otros, los cuales se caracterizan por implicar un movimiento que podría realizarse con desplazamiento en el mundo físico. Consideramos que esta matización también está conceptualizada en estos VMMIs, en forma de una TND potencial, cuya interacción con los indicadores de dirección podría activar una $T D$ y legitimar la construcción correspondiente. 


\section{3. "Verbo $+a$ " en el español}

En esta combinatoria también se han de tener en cuenta los verbos de $T D$ y los verbos de $T D$ y Manera, además de los tres tipos de verbos de Manera mencionados. El comportamiento de $a$ en los siguientes dos contextos apoya la afirmación de Fábregas de que $a$ es locativa, que en principio no codifica ni Dirección ni TD:

Por un lado, la co-ocurrencia entre el verbo y $a$ depende mucho del significado de Dirección del verbo:

Cuando se trata de verbo de $T D$, la generación de la TD entre este y a generalmente no implica dificultad: El niño subió al árbol. Esta observación también se aplica a los verbos de TD y Manera (Cifuentes, 2010; Pedersen, 2016), como se ejemplifica en (24):

(24) [huirse / precipitarse / escaparse / abalanzarse / trepar / refluir / afluir $]+a$.

La importancia del significado de Dirección también se observa en el siguiente grupo de verbos:

(25) a. ?El agua del río fluyó al mar.

b. El agua de la mesa refluyó al tubo.

c. Todo el mundo afluyó a la Puerta del Sol.

En (25a), el VMME fluir no implica Dirección y, según nuestra competencia como hablantes, resulta rara su combinatoria con $a$. No obstante, cuando la raíz verbal adquiere un prefijo ${ }^{10}$ direccional $r e$ - (25b) o $a$ - (25c), se convierte en un verbo de $T D \mathrm{y}$ Manera que codifica $T D$ y, en consecuencia, la combinatoria entre él y a se vuelve gramatical.

Por otro lado, cuando se trata de VMME (26a), VMMI (26b) o VME (26c), generalmente no cabe la combinación con $a$ :

(26) a. *[Flotó / navegó / cabalgó / callejeó / vagó / cojeó / renqueó / gateó] + al puerto.

10 Para los procesos de cambio léxico en estos verbos prefijados, véase Penas (2018). 
b. *Juan [tembló / se agitó / se estremeció / osciló / titubeó / tropezó / pataleó / cabeceó / pirueteó] + a la puerta.

c. *Juan [se rió / lloró / cantó / se enfadó] + a la puerta.

Si bien es cierto que un grupo reducido de VMMEs es compatible con $a$, esto debería ser tratado como caso excepcional del español (Martínez, 2001: 43-44). De acuerdo con el exhaustivo estudio de corpus de Pedersen (2016) sobre la combinatoria de "verbo de Manera $+a$ ", de entre los al menos 73 VMMEs tenidos en cuenta se encontraron 23 VMMEs que pueden darse con $a$, de los cuales, 11 VMMEs (27a) presentan más de 5 ejemplos de co-ocurrencia, mientras que los 12 VMMEs restantes (27b) registran menos de 5 ejemplos de co-ocurrencia:

(27) a. correr, saltar, volar, lanzarse, tirarse, arrojarse, caminar, fluir, montarse, andar, extenderse.

b. rodar, navegar, revolcarse, deslizarse, brincar, pasear, apresurarse, resbalarse, pisar, flotar, arrastrarse, conducir.

Cabe reiterar que dicho resultado proviene de un exhaustivo estudio de corpus. De hecho, según nuestra competencia como hablantes, la combinatoria entre $a \mathrm{y}$ algunos de los VMMEs expuestos en (27) se aprecia un tanto forzada para describir el evento de desplazamiento en el español.

De acuerdo con nuestra propuesta, si a fuera un indicador de dirección propiamente, debería poder darse con tantos VMMEs como sucede con hacia y hasta para generar la TD del evento de desplazamiento. La dependencia del significado direccional del verbo y la agramaticalidad de la combinatoria entre la mayoría de los VMMEs y $a$ (en contraste con la gramaticalidad entre la mayoría de los VMMEs y hacia / hasta), prueba que a es locativa y que no codifica Dirección.

Aunque estamos de acuerdo con Fábregas (2007) en que $a$ es locativa, conviene hacer una distinción entre nuestra propuesta y la suya.

Fábregas (2007) afirma que $a$ es locativa y argumenta que 1) los verbos de Manera que se dan con $a$ son VMMEs que codifican Trayectoria (TD en nuestra terminología), como volar, correr, caminar, etc.; 2) los que no se dan con $a$ son VMMIs 
que no la codifican, como flotar, temblar, bailar; 3) existe un grupo de verbos que son casos de variación, como gatear y cojear, que en principio son VMMIs, pero que para los hablantes que consideran aceptable su combinación con a codifican Trayectoria (TD en nuestra terminología).

Para nosotros, los verbos considerados por este autor como casos de variación son inherentemente VMMEs que sí codifican TND, ya que la realización de su acción verbal implica obligatoriamente un desplazamiento de la Figura en el mundo físico. Fábregas (2007: 185) apunta que los VMMEs como volar codifican Trayectoria (TD en nuestra terminología) ya que siempre implican el cambio de lugar, por lo tanto, la frase siguiente: \#Juan voló durante una hora y al terminar estaba en el mismo sitio suena rara, salvo que el sujeto se haya movido en círculos y haya vuelto al lugar de partida cuando terminó de volar. Sin embargo, cuando reemplazamos el verbo volar por los verbos conceptuados por él como casos de variación, la frase también denota el cambio de lugar de la Figura: \#Juan cojeó / gateó durante una hora y al terminar estaba en el mismo sitio. Aunque la acción verbal ocurriera en el supuesto caso de moverse en círculos, es decir, que la Figura volviera al lugar de partida después de realizar las acciones verbales de cojear o gatear en círculos, estas acciones verbales sí generarían una TND (o TD si se considera el movimiento en círculo como un tipo de dirección) en el espacio. Por lo tanto, a diferencia de Fábregas (2007), consideramos que los casos de variación sí son VMMEs que implican TND, y es la carencia de Dirección en a la que impide su co-ocurrencia con ellos.

En cuanto a los casos excepcionales - la agramaticalidad entre algunos VMMEs y $a$-, existen por lo menos dos posibles explicaciones de las que por limitaciones de espacio no nos ocupamos en este trabajo, aunque sí las mencionamos brevemente:

1) El español se halla en el proceso de desarrollo del patrón de marco satélite, y $a$ ha adquirido un significado direccional débil. Como afirma Martínez (2001), existe un contacto cada vez más estrecho entre el español y el inglés, ya que hay cada día más hablantes de español que utilizan el inglés en la vida cotidiana y están más expuestos a entornos anglófonos. Por lo tanto, cuando se ve influida por la estructura inglesa correspondiente, es lógico que la gramaticalidad de la combinatoria "VMME $+a$ " empiece por los "candidatos" más adecuados, es decir, los verbos de Manera menos 
marcada, ya que la generación entre los VMMEs que denotan una TND más fuerte y a implica un proceso relativamente más fácil para activar la $T D^{11}$.

2) Inferencia pragmática entre el VMME y la preposición locativa. La coocurrencia entre los VMMEs y las preposiciones locativas se observa en muchas lenguas (Cummins, 1996, para el italiano; Kopecka, 2009, para el francés; Gehrke, 2006, para el alemán; Nikitina, 2008, para el inglés; Tham, 2013, para el chino), independientemente de que estas rijan el patrón de marco verbal o el de marco satélite. Estos estudios muestran que la gramaticalidad de dicha combinatoria está relacionada con las propiedades del verbo (por ejemplo, los verbos que implican una TND más fuerte son más proclives a combinarse con las preposiciones locativas) o con componentes aspectuales del evento (por ejemplo, el desplazamiento puntual de menor distancia o duración de tiempo es más factible que se dé con las preposiciones locativas). Estos factores, entre otros, han favorecido la generación del significado direccional de la combinatoria de "VMME + preposición locativa" (Nikitina, 2008; Kopecka, 2009; Levin et al., 2009; Tham, 2013).

\section{Conclusiones}

En este trabajo hemos planteado que, en la combinatoria entre los verbos de movimiento y las preposiciones hacia, hasta y sus expresiones correspondientes chinas, la TND implicada en el verbo de Manera es primordial en la codificación del evento de desplazamiento, mientras que hacia y hasta y sus expresiones correspondientes chinas no aportan ningún tipo de Trayectoria y solo codifican Dirección. Por otra parte, el valor extensivo de hasta no favorece la generación de la TD del evento de desplazamiento cuando el verbo no implica ningún tipo de Trayectoria (TND o TD), mientras que el del marco preposicional chino yìzhí $+\mathrm{V}+$ dào 'hasta $+\mathrm{V}$ ' sí lo puede hacer.

11 Esta afirmación se corresponde con la de Pedersen (2016). Como hemos visto en el apartado 3.3, este autor concluye que cuanto más significado de direccionalidad implican los VMMEs, más posible será su combinación con $a$. Consideramos que los VMMEs no implican por sí mismos Dirección y que la direccionalidad de Pedersen coincide con la TND en nuestra propuesta, así que se podría reinterpretar su afirmación como sigue: cuanto más fuerte es el significado de TND implicado en los VMMEs, más posible será su combinación con $a$. 
Nuestra propuesta permite aportar luz a las discusiones más polémicas en las dos lenguas. En el chino, el comportamiento dependiente de dào 'llegar; a' -representante de los verbos de $T D$ que también pueden funcionar como $\mathrm{C} 2$ de la estructura serial-, de la TND implicada en el VMME precedente, muestra que solo funciona como indicador de meta que no puede aportar el significado de Trayectoria y, por lo tanto, la estructura serial a la que pertenece debería ser del tipo de lengua de marco satélite, en lugar de lengua de marco verbal o lengua de marco equipolente. En el español, la no aceptabilidad de "la mayoría de los VMMEs $+a$ ", en contraste con la gramaticalidad de "la mayoría de los VMMEs + hacia / hasta", apoya la afirmación de Fábregas (2007) de que $a$ es locativa, cuya carencia de Dirección ha impedido la generación de la TD en combinación con la TND implicada en los VMMEs.

En cuanto a la gramaticalidad de la combinatoria entre un grupo reducido de VMMEs y $a$, como hemos apuntado, podría deberse al proceso de desarrollo del patrón de marco satélite en el español y la adquisición del significado direccional de la preposición locativa $a$, o, a la inferencia pragmática entre el VMME y la preposición locativa.

Nuestra propuesta también permitiría ofrecer una explicación razonable sobre la coexistencia del patrón de lengua de marco satélite en el español. Aunque en el español rige preferentemente el patrón de lengua de marco verbal, no resulta imposible la existencia simultánea del patrón de lengua de marco satélite, ya que la combinatoria de "VMME + hacia / hasta" también es capaz de generar la TD de un evento de desplazamiento.

Por otra parte, planteamos que el predominio del patrón de lengua de marco verbal en el español habría inhibido en cierta medida el desarrollo del patrón de lengua de marco satélite, por lo que podemos encontrar VMMEs cuya combinación con hacia y hasta resulta de bajo perfil gramatical. La interacción entre la coexistencia del patrón de lengua de marco satélite y la inhibición ejercida por parte del patrón predominante de lengua de marco verbal, posibilita plasmar las características tipológicas del español en la codificación del evento de desplazamiento.

\section{Abreviaturas}


$\mathrm{C} 1$ : Constituyente 1 (de la estructura serial china)

C2: Constituyente 2 (de la estructura serial china)

DCM: Diccionario del Chino Moderno

ETT: Corpus "esTenTen18"

PA: partícula agentiva

PP: partícula perfectiva

SP: sintagma preposicional

TD: Trayectoria Direccional

TND: Trayectoria No Direccional

VME: verbo de Manera estática

VMME: verbo de Manera con movimiento externo

VMMI: verbo de Manera con movimiento interno 


\section{Bibliografía}

Aske, Jon (1989): "Path predicates in English and Spanish: A closer look", en: Proceedings of the Fifteenth Annual Meeting of the Berkeley Linguistics Society. Berkeley: University of California, 1-14.

Beavers, John/Levin, Beth/Tham, Shiao Wei (2010): “The typology of motion expressions revisited", en: Journal of Linguistics 46, 331-377.

Berthele, Raphael (2004): “The typology of motion and posture verbs: a varitionist account”, en: Bernd Kortmann (ed.): Dialectology meets Typology. Dialect Grammar from a Cross-linguistic Perspective. Berlin/New York: Mouton de Gruyter, 93-126.

Chen, Si Jia (2019): Estudio comparativo entre los patrones de lexicalización de los eventos de Movimiento en chino y en español. Tesis doctoral. Madrid: Universidad Complutense de Madrid.

Cifuentes Férez, Paula (2008): Motion in English and Spanish: A perspective from cognitive linguistics, typology, and psycholinguistics. Tesis doctoral. Murcia: Universidad de Murcia.

Cifuentes Férez, Paula (2010): "The semantics of the English and the Spanish motion verb lexicons", en: Review of Cognitive linguistics 8/2, 233-271.

Cuartero Otal, Juan (2006): “¿Cuántas clases de verbos de desplazamiento se distinguen en español?", en: Rilce: Revista de Filología Hispánica 22/1, 13-36.

Cummins, Sarah (1996): "Movement and Direction in French and English”, en: Toronto Working Papers in Linguistics 15. Toronto: University of Toronto, 31-54.

Demonte Barreto, Violeta (2011): "Los eventos de movimiento en español: construcción léxico-sintáctica y microparámetros preposicionales", en: Juan Cuartero Otal/Luis García Fernández/Carsten Sinner (eds.): Estudios sobre perifrasis y aspecto. München: Peniope, 16-42.

Dictionary Department of Institute of Linguistics of Chinese Academy of Social Sciences (2016): Xiandai hanyu cidian [Diccionario del Chino Moderno]. Beijing: The Commercial Press. [En el texto: DCM].

Fábregas, Antonio (2007): “An Exhaustive Lexicalization Account of Directional Complements", en: Language and Linguistics 34/2, 165-199. 
Fan, Li Ke (2013): Weiyi shijian de biaoda fangshi yanjiu - “yundong” yu "lujing”, "jufa hexin” yu "yiyi hexin” de hudong yu hezuo [Estudio sobre la expresión de los eventos de desplazamiento - Interacción y Colaboración entre "Movimiento" y “Trayectoria”, así como entre "el núcleo sintáctico” y “el núcleo semántico”]. Tesis doctoral. Shanghai: Universidad de Estudios Internacionales de Shanghai.

Gehrke, Berit (2006): "Putting Path in Place", en: Proceedings of Sinn und Bedeutung 11, 244-260.

Lamiroy, Béatriz (1991): Léxico y gramática del español: Estructuras verbales de espacio y de tiempo. Barcelona: Anthropos.

Levin, Beth (1993): English verb classes and alternations: a preliminary investigation. Chicago: University of Chicago Press.

Levin, Beth/Beavers, John/Tham, Shiao Wei (2009): “Manner of Motion Roots Across Languages: Same or Different?", presentado en: Word Formation from the Perspective of 'Core Lexical Elements'. Stuttgart: Universität Stuttgart.

Li, Feng Xiang (1997): “Cross-Linguistic Lexicalization Patterns: Diachronic Evidence from Verb - Complement Compounds in Chinese", en: Sprachtypol. Univ. Forsch. (STUF) 50/3, 229-252.

Kopecka, Anetta (2009): 'L'expresssion du déplacement en français: L'interaction des facteurs sémantiques, aspectuels et pragmatiques dans la construction du sens spatial", en: Langages 173, 54-77.

Martínez Vázquez, Montserrat (2001): “Delimited events in English and Spanish”, en: Estudios ingleses de la Universidad Complutense 9, 31-59.

Morimoto, Yuko (2001): Los verbos de movimiento. Madrid: Visor Libros.

Nikitina, Tatiana (2008): "Pragmatic factors and variation in the expression of spatial goals: The case of into vs. in", en: Anna Asbury/Jakub Dotlačil/Berit Gehrke/Rick Nouwen (eds.): Syntax and Semantics of Spatial P. Amsterdam: John Benjamins, 175-209.

Pedersen, Johan (2016): "Spanish Constructions of directed motion - a quantitative study: Typological variation and framing strategy", en: Stefan Th. Gries/Jiyoung Yoon (eds.): Corpus-based approaches to Construction Grammar. Amsterdam: John Benjamins, 95-144. 
Penas Ibáñez, María Azucena (2016): “La preposición: una categoría fronteriza entre la Semántica sintáctica y la Semántica léxica”, en: Benjamín GarcíaHernández/María Azucena Penas Ibáñez (eds.): Semántica latina y románica. Unidades de significado conceptual y procedimental. Bern: Peter Lang, 297-329.

Penas Ibáñez, María Azucena (2018): “Cambio semántico y lexemática verbal. De los preverbios latinos a los verbos españoles prefijados y de régimen preposicional. $\mathrm{Su}$ relación con los phrasal verbs", en: Romance Philology 72/2, 229-254.

Peyraube, Alain (2006): "Motion events in Chinese: A diachronic study of directional complements", en: Maya Hickmann/Stéphane Robert (eds.): Space in Languages: Linguistic Systems and Cognitive Categories. Amsterdam: John Benjamins, 121138.

Shi, Wen Lei (2014): Hanyu yundong shijian cihui leixing de lishi kaocha [Evolución del patrón de lexicalización de los eventos de desplazamiento en el chino]. Beijing, Commercial Press.

Slobin, Dan Isaac (2004): “The Many Ways to Search for a Frog: Linguistic Typology and the Expression of Motion Events", en: Sven Strömqvist/Ludo Verhoeven/Ruth Berman/Dan Slobin (eds.): Relating Events in Narrative, Vol. 2: Typological and Contextual Perspectives. Mahwah: Lawrence Erlbaum Associates, 219-257.

Tai, Hao-Yi James (2003): "Cognitive Relativism: Resultative Construction in Chinese", en: Language and Linguistics 4/2, 301-316.

Talmy, Leonard (1985): "Lexicalization patterns: Semantic structures in lexical forms", en: Timothy Shophen (ed.): Language Typology and Syntactic Description III: Grammatical Categories and the Lexicon. Cambridge: Cambridge University Press, 57-149.

Talmy, Leonard (1991): "Path to Realization: A Typology of Event Conflation", en: Proceedings of the Seventeenth Annual Meeting of the Berkeley Linguistics Society: General Session and Parasession on The Grammar of Event Structure. Berkeley: University of Califonia, 480-519.

Talmy, Leonard (2000): Toward a Cognitive Semantics, Vol. II: Typology and Process in Concept Structuring. Cambridge (MA): MIT Press.

Talmy, Leonard (2012): “Main verb properties”, en: International Journal of Cognitive Linguistics 3/1, 1-23. 
Tham, Shiao Wei (2013): "When motion and location yield direction: The case of Mandarin", en: Proceedings of the 37th Annual Meeting of the Berkeley Linguistics Society. Berkeley: University of California, 344-358.

Wälchli, Bernhard (2001): "A typology of displacement (with special reference to Latvian”, en: Sprachtypologie und Universalienforschung 54, 298-323.

Corpora empleados:

- Corpus de Davies

(Http://www.corpusdelespanol.org)

- Corpus "esTenTen 18 "

(https://app.sketchengine.eu/\#wordsketch?corpname=preloaded\%2Festenten 18 fl 5) 